\title{
A THOMSON'S PRINCIPLE FOR INFINITE, NONLINEAR RESISTIVE NETWORKS
}

\author{
L. DE MICHELE AND P. M. SOARDI
}

(Communicated by William D. Sudderth)

\begin{abstract}
Suppose $\Gamma$ is an infinite resistive electrical network with resistors of the form (2). We prove an existence and uniqueness theorem for the current generated by external current sources by establishing the analogue of Thomson's principle in suitable modular sequence spaces.
\end{abstract}

\section{INTRODUCTION}

An infinite resistive electrical network consists of an infinite, locally finite connected graph $\Gamma$ whose edges $B$ are assigned a relation

$$
F_{B}\left(v_{B}, i_{B}\right)=0
$$

between the voltage $v_{B}$ and the current $i_{B}$ flowing in $B$. We shall assume that the network is energized by external current sources represented by a 0 -chain. The voltage and current distributions in the network obey the classical Kirchhoff's laws.

When the relations (1) take the form: $v_{B}=i_{B} r_{B}$ (where $r_{B}$ is the resistance of the edge $B$ ), the network is called linear or ohmic. The theory of infinite linear networks was founded by $\mathrm{H}$. Flanders $[\mathrm{F}]$, who proved that, when the number of energy sources is finite, there exists a current of finite energy flowing in the network, and that such a current is unique provided it satisfies the extra condition of being a limit of finite currents. The case of infinitely many sources was considered by Zemanian [Z1, Theorem 3.3], always under the assumption of finite total power.

In this paper we study nonlinear networks where the relations (1) take the form

$$
v_{B}=\operatorname{sign}\left(i_{B}\right) p_{B}\left(\left|i_{B}\right|\right),
$$

where $p_{B}$ is a continuous strictly increasing function such that $p_{B}(0)=0$ (and satisfying a technical condition, see $(4 b))$.

Received by the editors June 23, 1989 and, in revised form, September 20, 1989.

1980 Mathematics Subject Classification (1985 Revision). Primary 94C15; Secondary 46N05.

Key words and phrases. Nonlinear, infinite electrical networks, flows, modular sequence spaces, minimum energy, currents. 
Finite nonlinear networks of this kind were considered in the pioneering papers by Duffin [Du] and Minty [M]. Infinite nonlinear (not necessarily resistive) networks were studied in great generality by Dolezal [D1, D2] and by Dolezal and Zemanian [D-Z] in the context of Hilbert spaces (and total finite power).

As noted by Zemanian, Kirchhoff's laws alone, coupled with the relationships between current and voltage distributions given by the elements of the network, are in general too weak to yield a unique solution (cf. the papers [Z2, Z3, Z4]; see also, in this context, $\S 4$ in Soardi and Woess [S-W]). In the papers mentioned in the previous paragraphs the natural approach is taken of restricting the class of admissible solutions to the class of all currents of finite energy. Accordingly, the right mathematical setting is provided by the Hilbert space. However, in nonlinear resistive networks where the resistors have the form (2), the finite energy restriction would impose too strong conditions on the functions $p_{B}$.

Our starting point is the illuminating discussion in Thomassen's paper [T] of currents in finite monotone networks. The role of the energy of a 1-chain $I=\sum_{B \in E(\Gamma)} i_{B} B$ is played by the functional

$$
\boldsymbol{W}(I)=\sum_{B \in E(\Gamma)} \int_{0}^{\left|i_{B}\right|} p_{B}(t) d t .
$$

Note that in the linear case $W(I)$ is exactly one-half the power dissipated by $I$ (or the energy of $I$ ). It turns out that the right mathematical framework in the nonlinear resistive case (2) is the theory of modular sequence spaces, a generalization of Orlicz sequence spaces investigated by Woo [W].

In $\S \S 2$ and 3 we fix the notation and give a short account of the relevant properties of the modular sequence spaces. In $\S 4$ we prove our main result, a Thomson's principle for nonlinear resistive networks. Namely, we show that, under natural assumptions on the external current sources, there is a unique flow $I^{0}$ which minimizes $\boldsymbol{W}$ among all flows belonging to the modular sequence space. Such a flow is the unique 1-chain (in such a space) which satisfies Kirchhoff's node and loop laws (in a strenghtened form: see Remark 2).

\section{Notation}

Let $\Gamma$ denote an oriented, infinite, locally finite, connected graph with no multiple edges and no self-loops. We denote by $V(\Gamma)$ the vertex set and by $E(\Gamma)$ the (oriented) edge set. No result presented here will depend on the orientation chosen for the edges of $\Gamma$.

Following Flanders [F], a 0-chain

$$
l=\sum_{x \in V(\Gamma)} l_{x} x
$$

and a 1-chain

$$
I=\sum_{B \in E(\Gamma)} i_{B} B
$$


are defined as formal, real, infinite linear combinations of vertices $x$ or of edges $B$, respectively.

In the sequel we agree that, whenever $I, J, \ldots, Z$ denote 1-chains, $i_{B}, j_{B}$, $\ldots, z_{B}$ will denote the corresponding coefficients of the edge $B$.

The local finiteness of $\Gamma$ allows us to define the boundary $\partial I$ of a 1-chain $I$ as the 0 -chain

$$
\partial I=\sum_{B \in E(\Gamma)} i_{B}(y(B)-x(B))
$$

where $x(B)$ and $y(B)$ are the initial and the terminal point of $B$, respectively. A 1 -chain $Z$ is called a cycle if $\partial Z=0$.

Next, we fix the assumptions on the functions $p_{B}$ in (2).

(4a) For every $B \in E(\Gamma), p_{B}$ is a continuous strictly increasing function on $[0,+\infty)$, and $p_{B}(0)=0$.

(4b) There exist a number $r$ and a finite set $\mathscr{F} \subset E(\Gamma)$ such that

$$
\frac{t p_{B}(t)}{M_{B}(t)} \leq r \quad \text { for all } t \in\left(0, a_{B}\right) \text { and } B \notin \mathscr{F}
$$

where

$$
M_{B}(t)=\int_{0}^{t} p_{B}(u) d u
$$

and $a_{B}$ is the unique positive number such that $M_{B}\left(a_{B}\right)=1$.

The technical condition (4b) is equivalent to the uniform $\Delta_{2}$ condition in [W, p. 273] for the functions $N_{B}(t)$ defined as

$$
N_{B}(t)=M_{B}\left(a_{B} t\right) .
$$

Observe that $M_{B}$ is a strictly convex function and that (4b) implies $M_{B}(t) \geq$ $\left(t / a_{B}\right)^{r}$ for $0 \leq t \leq a_{B}$ and $B \notin \mathscr{F}$ (compare with [W, Proposition 3.2]).

The functional $\boldsymbol{W}$ becomes

$$
\boldsymbol{W}(I)=\sum_{B \in E(\Gamma)} M_{B}\left(\left|i_{B}\right|\right) .
$$

Let $p_{B}^{-1}$ denote the inverse function of $p_{B}$ and set

$$
M_{B}^{*}(t)=\int_{0}^{t} p_{B}^{-1}(u) d u
$$

Then $M_{B}$ and $M_{B}^{*}$ are complementary Orlicz functions and the following simple relations hold for all $a, b>0$ [K-R, pp. 12-13].

$$
\begin{gathered}
a b \leq M_{B}(a)+M_{B}^{*}(b) \quad \text { (Young's inequality) } \\
a p_{B}(a)=M_{B}(a)+M_{B}^{*}\left(p_{B}(a)\right) .
\end{gathered}
$$




\section{MOdUlar SEQUENCE SPACES AND KIRCHHOFF'S LAWS}

Our reference for modular sequence spaces is the above quoted paper by Woo and the book by Lindenstrauss and Tzafriri [L-T]. The standard results in functional analysis used below can be found in Dunford and Schwartz [D-S].

Let the countable set of functions $\left\{p_{B}\right\}$ satisfy assumptions (4a) and (4b) of $\S 2$. The modular sequence space $\ell\left\{M_{B}\right\}$ is defined as the linear space of all 1-chains $I$ such that, for some $t>0$

$$
\sum_{B \in E(\Gamma)} M_{B}\left(\frac{\left|i_{B}\right|}{t}\right)<\infty
$$

For all $I \in \ell\left\{M_{B}\right\}$ set

$$
\|I\|=\inf \left\{t: \sum_{B \in E(\Gamma)} M_{B}\left(\frac{\left|i_{B}\right|}{t}\right) \leq 1\right\} .
$$

With this norm $\ell\left\{M_{B}\right\}$ becomes a Banach space. We denote by $c\left\{M_{B}\right\}$ the closed linear subspace of all 1-chains $I$ such that (7) holds for every $t>0$. Since, by (4b), the uniform $\Delta_{2}$ condition is satisfied by the normalized sequence $\left\{N_{B}\right\}_{B \in E(\Gamma)}, \ell\left\{N_{B}\right\}=c\left\{N_{B}\right\}$ and the elementary 1-chains $\{B\}_{B \in E(\Gamma)}$ form an unconditional basis [W, Theorems 3.5 and 4.4].

Woo's results on modular sequence spaces are stated in terms of the normalized spaces $\ell\left\{N_{B}\right\}$ for technical reasons. However, it is easy to prove (see the proof of the Proposition below), that $\ell\left\{M_{B}\right\}$ is isometrically isomorphic to $\ell\left\{N_{B}\right\}$, so that they have the same good topological properties. In particular, if we denote, as usual, by $X^{*}$ the dual of a Banach space $X$ and let $\cong$ denote isometric isomorphism, we have the following results.

\section{Proposition.}

$$
\begin{gathered}
\ell\left\{M_{B}\right\}=c\left\{M_{B}\right\}, \\
c\left\{M_{B}^{*}\right\}^{*} \cong \ell\left\{M_{B}\right\}, \\
\ell\left\{M_{B}\right\}^{*} \cong \ell\left\{M_{B}^{*}\right\},
\end{gathered}
$$

and the duality is expressed by the pairing

$$
\langle I, J\rangle=\sum_{B \in E(\Gamma)} i_{B} j_{B} \quad I \in \ell\left\{M_{B}\right\}, J \in \ell\left\{M_{B}^{*}\right\} .
$$

Proof. Let $\boldsymbol{T}$ denote the fcllowing linear transformation on the linear space of all 1-chains

$$
\boldsymbol{T}\left(\sum_{B \in E(\Gamma)} i_{B} B\right)=\sum_{B \in E(\Gamma)} i_{B} a_{B}^{-1} B
$$

and let $\boldsymbol{T}_{\ell\left\{M_{B}\right\}}$ denote the restriction to $\ell\left\{M_{B}\right\}$. Then, $\boldsymbol{T}_{\ell\left\{M_{B}\right\}}$ is an isometric isomorphism of $\ell\left\{M_{B}\right\}$ onto $\ell\left\{N_{B}\right\}$, mapping $c\left\{M_{B}\right\}$ onto $c\left\{N_{B}\right\}$. Hence (8a) holds (see above). 
Now observe that the proof of Proposition 4.1 and of the first statement in Theorem 4.2 in [W] depend neither on normalization nor on uniform $\Delta_{2}$ condition. Therefore $(8 \mathrm{~b})$ follows from these results applied to the complementary functions $M_{B}^{*}$ and $M_{B}$. The same argument and (8a) give (8c) and (9).

Remark 1 . Note that (8a) implies that the $W(I)$ is finite for every $I \in \ell\left\{M_{B}\right\}$.

The pairing (9) induces the following equivalent norm on $\ell\left\{M_{B}\right\}$

$$
\|I\|=\sup \left\{\sum_{B \in E(\Gamma)}\left|i_{B} j_{B}\right|: \sum_{B \in E(\Gamma)} M_{B}^{*}(J) \leq 1\right\}
$$

(see [W, Proposition 4.1]).

Lemma 1. Let $I=\sum_{B \in E(\Gamma)} i_{B} B$ belong to $\ell\left\{M_{B}\right\}$. Then the 1-chain $H=$ $\sum_{B \in E(\Gamma)} p_{B}\left(\left|i_{B}\right|\right) B$ belongs to $\ell\left\{M_{B}^{*}\right\}$.

Proof. By (6), (4b) and the definition of $a_{B}$, we have that, for all $B \notin \mathscr{F}$, $\left|i_{B}\right|<a_{B}$ implies

$$
M_{B}^{*}\left(p_{B}\left(\left|i_{B}\right|\right)\right) \leq\left|i_{B}\right| p_{B}\left(\left|i_{B}\right|\right) \leq r M_{B}\left(\left|i_{B}\right|\right) .
$$

Since by $(8 \mathrm{a})$

$$
\sum_{B \in E(\Gamma)} M_{B}\left(\left|i_{B}\right|\right)<+\infty
$$

by (4a) and the definition of $a_{B}$ again, for all but a finite number of edges $B$, $\left|i_{B}\right|<a_{B}$. The thesis follows from (11).

Definition. Taking the above Proposition and Lemma 1 into account, we define the resistance operator $\boldsymbol{R}$ from $\ell\left\{M_{B}\right\}$ to $\ell\left\{M_{B}\right\}^{*}$ as

$$
\boldsymbol{R}(I)=\sum_{B \in E(\Gamma)} \operatorname{sign}\left(i_{B}\right) p_{B}\left(\left|i_{B}\right|\right) B, \quad I \in \ell\left\{M_{B}\right\} .
$$

In the following we will denote by $\mathscr{Z}$ the linear subspace of all cycles belonging to $\ell\left\{M_{B}\right\}$.

Lemma 2. $\mathscr{Z}$ is a closed subspace of $\ell\left\{M_{B}\right\}$.

Proof. For all $x \in V(\Gamma)$, let $E_{x}$ denote the finite subset of edges having $x$ as an extreme. For $B \in E_{x}$ define $\phi_{B}(x)$ to be 1 or -1 according to $B=[y, x]$ or $B=[x, y]$. One checks immediately that for every 1-chain $Z$ (cf. (9))

$$
\partial Z=\sum_{x \in V(\Gamma)}\left(\sum_{B \in E_{x}} \phi_{B}(x) z_{B}\right) x=\sum_{x \in V(\Gamma)}\langle\Phi(x), Z\rangle x
$$

where $\Phi(x)=\sum_{B \in E_{x}} \phi_{B}(x) B \in \ell\left\{M_{B}^{*}\right\}$ for all $x$. The thesis follows by (13) and by Lemma 1 .

Let $l=\sum_{x \in V(\Gamma)} l_{x} x$ be a 0 -chain with the property that there exists $L \in$ $\ell\left\{M_{B}\right\}$ such that $\partial L=\imath$. For instance this is certainly the case if $l$ is a finite chain and $\sum_{x \in V(\Gamma)} l_{x}=0$ (see e.g [F, p. 328]). 
Definition. We say that a 1 -chain $I \in \ell\left\{M_{B}\right\}$ satisfies Kirchhoff's laws if

$$
\partial I+i \equiv 0 \quad \text { (node law) }
$$

In particular, a 1-chain in $\ell\left\{M_{B}\right\}$ satisfying (14) is called a flow. A flow satisfying also (15) is called a current generated by $l$.

Remark 2. Equation (15) expresses the fact that the voltage drop is zero along all cycles in $\ell\left\{M_{B}\right\}$, finite or infinite. This strengthened form of Kirchhoff's loop law was formulated by Zemanian [Z1] in Hilbert spaces, and appears to be better adapted to infinite networks. In general this formulation is strictly stronger than that of requiring zero voltage drop only along finite cycles.

\section{THOMSON'S PRINCIPLE}

In this section we prove our main result announced in $\S 1$.

Theorem. Let $\Gamma$ and the functions $p_{B}$ be as above. Suppose that $l=\partial L$ for some $L \in \ell\left\{M_{B}\right\}$. Then, there exists a unique current $I^{0} \in \ell\left\{M_{B}\right\}$ generated by $l$, and $I^{0}$ is the unique minimum point of $\boldsymbol{W}$ over all flows in $\ell\left\{M_{B}\right\}$.

Proof. Let

$$
\mathscr{X}=-L+\mathscr{Z}, \mathscr{X}_{n}=\{I \in \mathscr{X}:\|I\| \leq n\} .
$$

Note that $\mathscr{X}$ is nonempty and $I \in \mathscr{X}$ if and only if $I$ is a flow. For the reader's convenience we split the proof in several steps.

1. $W$ is a strictly convex functional on $\ell\left\{M_{B}\right\}$. In fact, for all $B$, all 1-chains $I, J$ in $\ell\left\{M_{B}\right\}$, and every $\lambda \in(0,1)$ we have

$$
\begin{aligned}
M_{B}\left(\left|\lambda i_{B}+(1-\lambda) j_{B}\right|\right) & \leq M_{B}\left(\lambda\left|i_{B}\right|+(1-\lambda)\left|j_{B}\right|\right) \\
& <\lambda M_{B}\left(\left|i_{B}\right|\right)+(1-\lambda) M_{B}\left(\left|j_{B}\right|\right)
\end{aligned}
$$

whence the assertion.

2. $W$ is weak-star lower sequentially semicontinuous on $\ell\left\{M_{B}\right\}$. First recall that, by (8b), $\ell\left\{M_{B}\right\}$ is the dual space of $c\left\{M_{B}^{*}\right\}$. Let $\left\{I^{(k)}\right\} \subset \ell\left\{M_{B}\right\}$ be a sequence converging to $I$ in the weak-star topology. Then $I^{(k)}$ converges to $I$ coordinatewise, i.e.

$$
i_{B}^{(k)} \rightarrow i_{B} \quad \text { as } \quad k \rightarrow \infty \quad \text { for all } B \in E(\Gamma) .
$$

By Fatou's lemma

$$
\begin{aligned}
\liminf _{k \rightarrow \infty} \mathbf{W}\left(I^{(k)}\right) & =\liminf _{k \rightarrow \infty} \sum_{B \in E(\Gamma)} M_{B}\left(\left|i_{B}^{(k)}\right|\right) \\
& \geq \sum_{B \in E(\Gamma)} \lim _{k \rightarrow \infty} M_{B}\left(\left|i_{B}^{(k)}\right|\right) \\
& =\boldsymbol{W}(I) .
\end{aligned}
$$


3. $W$ is everywhere Gateaux differentiable with $G$-differential

$$
W^{\prime}(I) Z=\sum_{B \in E(\Gamma)} \operatorname{sign}\left(i_{B}\right) p_{B}\left(\left|i_{B}\right|\right) z_{B}
$$

for all $Z \in \ell\left\{M_{B}\right\}$.

We have to prove that

$$
\lim _{t \rightarrow 0} \frac{\mathbf{W}(I+t Z)-\mathbf{W}(I)}{t}=\sum_{B \in E(\Gamma)} \operatorname{sign}\left(i_{B}\right) p_{B}\left(\left|i_{B}\right|\right) z_{B} .
$$

Now, for every $B$,

$$
\lim _{t \rightarrow 0} \frac{M_{B}\left(\left|i_{B}+t z_{B}\right|\right)-M_{B}\left(\left|i_{B}\right|\right)}{t}=\operatorname{sign}\left(i_{B}\right) p_{B}\left(\left|i_{B}\right|\right) z_{B} .
$$

Moreover, for $|t| \leq 1$

$$
\left|\frac{M_{B}\left(\left|i_{B}+t z_{B}\right|\right)-M_{B}\left(\left|i_{B}\right|\right)}{t}\right| \leq\left|z_{B}\right| p_{B}\left(\left|i_{B}\right|+\left|z_{B}\right|\right),
$$

and $\sum_{B \in E(\Gamma)}\left|z_{B}\right| p_{B}\left(\left|i_{B}\right|+\left|z_{B}\right|\right)<\infty$ by Lemma 1, (8c), and (9). Therefore (16) follows from Lebesgue dominated convergence theorem.

4. There exists a unique $I^{0} \in \mathscr{X}$ which minimizes $W$ on $\mathscr{X}$. By the definition of $\|\cdot\|$ and (5)

$$
\begin{aligned}
\|I\| & =\sup \left\{\sum_{B \in E(\Gamma)}\left|i_{B} j_{B}\right|: \sum_{B \in E(\Gamma)} M_{B}^{*}\left(\left|j_{B}\right|\right) \leq 1\right\} \\
& \leq 1+\boldsymbol{W}(I),
\end{aligned}
$$

so that $W(I) \rightarrow+\infty$ as $\|I\| \rightarrow+\infty$.

Hence, there exists $n$ such that

$$
\alpha=\inf \{\mathbf{W}(I): I \in \mathscr{Z}\}=\inf \left\{\mathbf{W}(I): I \in \mathscr{X}_{n}\right\} .
$$

Clearly $\mathscr{X}_{n}$ is compact in the weak-star topology. As noted in [W, p. 273], $c\left\{M_{B}^{*}\right\}$ is separable, so that the weak-star topology in $\mathscr{X}_{n}$ is also metrizable. By Step 2 there exists $I^{0} \in \mathscr{X}_{n}$ such that $\alpha=W\left(I^{0}\right)$ and, by Step $1, I^{0}$ is the unique minimum point of $W$ on $\mathscr{Z}$ with this property.

5. $I^{0}$ is the unique current generated by $l$. Since $I^{0}$ minimizes $\boldsymbol{W}$ on $\mathscr{X}$, then

$$
W^{\prime}\left(I^{0}\right) Z=0 \quad \text { for all } Z \in \mathscr{Z}
$$

so that $I^{0}$ satisfies Kirchhoff's loop law by (16). Since $I^{0}$ is the unique minimum point of $W$ on $\mathscr{X}$, by [V, Theorem 9.1], applied to the linear manifold $\mathscr{X}, I^{0}$ is also the unique element in $\mathscr{X}$ satisfying (17).

This concludes the proof. 


\section{REFERENCES}

[D1] V. Dolezal, Hilbert networks, SIAM J. Control Optim. 12 (1974), 755-778.

[D2] _ Generalized Hilbert networks, SIAM J. Control Optim. 14 (1976), 26-41.

[D-Z] V. Dolezal and A. H. Zemanian, Hilbert networks II: Some qualitative properties, SIAM J. Control Optim. 13 (1975), 153-161.

[Du] R. J. Duffin, Nonlinear networks IIa, Bull. Amer. Math. Soc. 53 (1947), 963-971.

[D-S] N. Dunford and J. T. Schwartz, Linear operators, Part I, Wiley Interscience, New York, 1958.

[F] H. Flanders, Infinite networks I-Resistive networks, IEEE Trans. Circuit Theory 18 (1971), 326-331.

[K-R] M. G. Krasnoselskii and Y. B. Rutickii, Convex functions and Orlicz spaces, Nordhoff, Groningen, 1961.

[L-T] J. Lindenstrauss and L. Tzafriri, Classical Banach spaces part I, Sequence spaces, SpringerVerlag, Berlin, Heidelberg, New York, 1977.

[M] G. J. Minty, Monotone networks, Proc. London Math. Soc. 257 (1960), 194-212.

[S-W] P. M. Soardi and W. Woess, Uniqueness of currents in infinite electrical networks, Università di Milano, 1988, Discrete Appl. Math., in print.

[T] C. Thomassen, Resistances and currents in infinite electrical networks, J. Combin. Theory Ser. B, in print.

[V] M. M. Vainberg, Variational method and the method of monotone operators in the theory of nonlinear equations, Wiley Interscience, New York, 1973.

[W] J. Y. T. Woo, On modular sequence spaces, Studia Math. 48 (1973), 271-289.

[Z1] A. H. Zemanian, Infinite electrical networks, Proc. IEEE 64 (1976), 6-17.

[Z2] _ The complete behaviour of certain infinite networks under Kirchhoff's loop and node law, SIAM J. Appl. Math. 30 (1976), 278-295.

[Z3] _ Countably infinite, nonlinear time-varying active electrical networks, SIAM J. Math. Anal. 10 (1979), 944-960.

[Z4] _ Nonlinear, resistive, countably infinite electrical networks, Applicable Anal. 8 (1978), 185-192.

Dipartimento di Matematica, Università di Milano via Saldini 50, 20133 Milano, Italy 